\title{
A novel productivity evaluation approach based on the morphological analysis and fuzzy mathematics: insights from the tight sandstone gas reservoir in the Ordos Basin, China
}

\author{
Shanyong Liu ${ }^{1,2,3} \cdot$ Gongyang Chen ${ }^{2} \cdot$ Yishan $\mathrm{Lou}^{4} \cdot$ Liang $\mathrm{Zhu}^{5} \cdot$ Daoyao $\mathrm{Ge}^{2}$
}

Received: 19 September 2019 / Accepted: 13 December 2019 / Published online: 27 December 2019

(c) The Author(s) 2019

\begin{abstract}
Tight gas reservoirs have rich potential resources, which are hot spots in unconventional oil and gas exploration and development. Due to their strong heterogeneity and complex pore structures, the conventional approaches of productivity evaluation always have difficulty in predicting the gas content. This study aims to devise a new method to interpret the productivity of LX Block in the Ordos Basin using the morphological theory and fuzzy mathematics. First, core test results were used to investigate the reservoir quality and physical properties. Then, the change law of gas content was defined by the morphological theory of logging and mud logging curves. Assignments of those factors that affected the final production were provided based on fuzzy mathematics. Finally, the prediction model of productivity was established. The results show that the lower limit of the reservoir thickness in the LX Block is $3.1 \mathrm{~m}$, whereas the porosity and permeability are $5 \%$ and $0.15 \times 10^{-3} \mu \mathrm{m}^{2}$, respectively. The morphological characteristic of the gas logging curve for those layers with high potential production normally presents a box shape with a high relative number of serration. The reservoir in the studied area can be classified into four categories according to the relationship between the logging curve shape and daily production, and each category is automatically identified. The coincidence rate between the prediction results and the gas test results is $84.1 \%$, which satisfies the demand on the field. The findings have important theoretical and practical significance for screening the location of fracturing spots and predicting the production of tight gas reservoirs.
\end{abstract}

Keywords Tight gas $\cdot$ Productivity evaluation $\cdot$ Morphological analysis $\cdot$ Gas logging curve method $\cdot$ Fuzzy mathematics

Shanyong Liu

liushanyong@yangtzeu.edu.cn

1 The Key Laboratory of Well Stability and Fluid \& Rock Mechanics in Oil and Gas Reservoir of Shaanxi Province, Xi' an Shiyou University, Xi' an 710065, Shaanxi, China

2 Institute of Mud Logging Technology and Engineering, Yangtze University, Jingzhou 434023, Hubei, China

3 Shandong Provincial Key Laboratory of Depositional Mineralization and Sedimentary Mineral, Shandong University of Science and Technology, Qingdao 266590, Shandong, China

4 College of Technology and Engineering, Yangtze University, Jingzhou 434020, Hubei, China

5 School of Petroleum Engineering, Yangtze University, Wuhan 430100, Hubei, China

\section{Introduction}

Global oil and gas resources can be grouped into two categories: conventional and unconventional resources, among which unconventional resources account for approximately $80 \%$ of the total amount. As one of the most important unconventional resources, tight gas is a hot field of exploration and development worldwide (Kulga et al. 2017). In 2012 , the output of natural gas in the USA was approximately $7132 \times 10^{12} \mathrm{~m}^{3}$, of which the production of tight gas was approximately $1800 \times 10^{12} \mathrm{~m}^{3}$, accounting for $26 \%$ of the total amount. In the same period, the corresponding data for China were $1070 \times 10^{12} \mathrm{~m}^{3}$ total and $265 \times 10^{12} \mathrm{~m}^{3}$ for tight gas (Zou et al. 2015). Tight gas reservoirs are widely present in China; there are more than ten basins including Ordos, Sichuan, Shanxi, Songliao, and Tarim with extremely rich resource potential (Wu et al. 2017a, b). However, it is also accompanied by the complex geological conditions, poor reservoir physical properties and strong reservoir 
heterogeneity (Shao et al. 2017). Hydraulic fracturing must be implemented to obtain commercial gas production. Hence, it is necessary to make an accurate forecast for the reservoir productivity before fracturing, which has a significant effect on the formation screening, treatment optimization and production allocation (Liu et al. 2018a, b). At present, logging interpretation (LI) is the main method for gas identification and evaluation based on the relationship of the log response (Anand 2017). However, LI is inappropriate for tight gas reservoirs due to the restriction of the resolution ratio, complex relationship of the log response and low resistivity of the gas layer (Sarhan et al. 2017). The gas logging interpretation (GLI), which collects the data signal from the well and has the advantages of rapidity and directness, is another method to determine the gas content. However, the absolute value of gas logging data in a tight gas reservoir is lower than that in a conventional reservoir, so it is difficult to acquire all gas component data, which causes a large difference between actual production and forecast production (Fang et al. 2018). In addition, varieties of mathematical algorithms are introduced to the evaluation models for accurate forecasting. Neuro-fuzzy inference system was adopted in the determination of heterogenous sedimentary rocks which could be confidently used in the field of geotechnical engineering (Umrao et al. 2018). An improved analytic hierarchy process was proposed to evaluate the fracability of tight sandstone reservoirs in the Junggar Basin (Song et al. 2019). Some scholars come up with an unsupervised machine learning approach on the study of the reservoir quality with artificial neural network (ANN) classification (Abdelmoneam et al. 2019).

Generally, either LI or GLI can achieve good evaluation results for conventional sand reservoirs. For unconventional reservoirs such as tight gas, it has difficulty in productivity evaluation because of the complex pore structures and percolation mechanism. The LX Block is located in the Jinxi flexural fold belt on the eastern margin of the Ordos Basin, which is connected with the Northern Shanxi slope in the west and Lvliang uplift in the east. The tight gas resources in this area, which average porosity and permeability are $7.7 \%$ and $1.7 \times 10^{-3} \mu \mathrm{m}^{2}$, respectively, are nearly $6.4 \times 10^{12} \mathrm{~m}^{3}$. However, there have big differences of gas testing results among different wells with different layers, especially after hydraulic fracturing. Therefore, it is urgent to study a new method for gas productivity evaluation of tight sandstone gas.

\section{State of the art}

Scholars worldwide have conducted in-depth studies on the productivity forecast with various methods such as logging interpretation, mud logging interpretation (MLI) and lab core tests (Schmitt et al. 2015; Montgomery and O'Sullivan 2017; Sakhaee-Pour 2017; Chaki et al. 2018). The characteristics of the gas content of conventional and unconventional reservoirs have been contrasted and analyzed by Liu and Sun based on the LI and MLI methods (Sun 2013; Liu et al. 2018a, b). The scope of the logging evaluation was expanded, and a comprehensive evaluation method was proposed based on six properties: physical property, electrical property, oil-gas-bearing property, hydrocarbon source rock characteristics, rock brittleness and in situ stress anisotropy. This approach can qualitatively evaluate the productivity potential of unconventional reservoirs but cannot quantitatively describe the gas-bearing characteristics of reservoirs. Liu improved the gas logging curve method and found a strong correlation between the ratio of total hydrocarbon to the background value and the productivity (Liu et al. 2016). The results had good application effects for specific wells, but it also had an obvious regional limitation. Wu built a real-time evaluation system based on the logging-while-drilling (LWD) data and mud logging data, which could make a quick identification on oil and gas layers (Wu et al. 2017a, b). The forecast results were sufficiently good under the condition of data integrity, but this integrity occurred less frequently in the field due to the cost limits. None of the above interpretation methods consider the effects of engineering factors such as perforating, fracturing and other stimulation approaches, which is most important for the enhancement of the gas production.

Experimental testing is another method to evaluate the gas content of a reservoir, which includes scanning electron microscopy (SEM), nuclear magnetic resonance (NMR), and permeability testing (Guo et al. 2015; Fall et al. 2015). The porosity and permeability obtained using these methods are plotted in the same coordinate system, and the lower limit values of the reservoir properties can be identified. According to the relationship between the oil test conclusion and the lower limit values of reservoir physical properties, the target zone can be easily selected. Xiao et al. (2017) analyzed the fluid distribution characteristics in rock pores with NMR, and the quantitative criteria of the porosity and reservoir classification were established. Sander et al. (2017) determined the lower limits of reservoir physical properties with the unsteady permeability test method. Good results were obtained through field application. The experimental method is widely used in the productivity evaluation, and the test results are accurate. However, the cost of the lab test is very high, and the test results cannot reflect the physical properties of the entire reservoir, which leads to an obvious deviation for the strong heterogeneous gas reservoir.

Although the productivity evaluation of tight sand gas has been deeply studied, limitations remain. Those approaches are mainly based on their own objectives and experimental means, which lack comprehensive study on the productivity 
prediction. Therefore, a new method of production evaluation in tight sandstone gas reservoirs is urgently required. This study combines the field data and core lab test results to evaluate the reservoir quality in the LX Block. Then, we analyze the causes for the variation in the logging curve shape and determine the quantitative relation between the gas logging cure shape and the productivity. Taking the fracturing technology of tight sand gas into consideration, the rock brittleness is set as the key engineering parameter, and the fuzzy mathematical method is used to assign those key parameters. Finally, the productivity prediction model is established based on the method of multiple linear regression.

The remainder of this paper is organized as follows. The assessment methodology under mud logging and well logging for tight gas is studied in "Methodology" section. The new model is applied to the LX Block, and the results are discussed in "Results analysis and discussion" section. The conclusions are summarized in "Conclusion" section.

\section{Methodology}

\section{Reservoir evaluation on the LX Block}

\section{Study on the petrological characteristics}

The tectonic movement in the western part of the LX Block is weak; faults and local structures are not developed. As shown in Table 1, from the Taiyuan formation to the Shiqianfeng formation, the quartz content gradually decreases, while the feldspar content increases, and the cutting content decreases from the Shanxi formation to the Shiqianfeng formation. On the whole, the reservoir in the LX Block is mainly composed of clastic rocks, and the clastic components are mainly quartz, feldspar and rock debris (Fig. 1).

\section{Study on the physical characteristics}

The reservoir physical properties reflect the reservoir capacity and fluid percolation ability, which is mainly manifested as porosity and permeability (Cao and Lei 2019). According to 2215 data points from 15 wells, the reservoir porosity in the LX area is mainly distributed in the range of $0.3-23.5 \%$ with an average porosity of $7.72 \%$ and a main porosity peak of $5.0-10.8 \%$. Due to the data missing for permeability, we collect fewer data points and the average permeability was $1.7 \times 10^{-3} \mu \mathrm{m}^{2}$, and $83 \%$ of the samples have permeability below $1.0 \times 10^{-3} \mu \mathrm{m}^{2}$ (see Fig. 2).

\section{Study on the pore structure characteristics}

The pore structure is very important to the physical properties of the reservoir. The SU8010 Ultra-High Resolution $(1.0 \mathrm{~nm})$ Scanning Electron Microscope had been used to make a thin section analysis of core samples from the study area; we found diverse pore types in the study area. As shown in Fig. 3, there are three main types of pores in the research area: (1) intergranular pores, which have good pore connectivity and are developed in all layers, but they are not large in scale and are very unevenly distributed with strong heterogeneity; (2) intragranular pores, whose formation is early and widespread, but the width is narrow; and (3) microfractures, including intergranular cracks and fractures, which are a type of secondary pore formed by the fracture of external forces.

\section{Study on the reservoir quality}

To obtain the lower limit of the reservoir properties, reservoir quality has been studied. The effective thickness of gas reservoir has a positive correlation with productivity, and its lower limit in LX Block is considered to be $7.0 \mathrm{~m}$. In addition, to determine the lower limit of the physical properties, the gas testing method is used. For the test stages, the equalization treatment method of each layer was adopted for the porosity and permeability data; after recounting the test data of 18 wells, we found that the minimum porosity and minimum permeability showed a gradual increase from gas free layer to gas layer. When the porosity is above $5.2 \%$ and the permeability is above $0.15 \times 10^{-3} \mu \mathrm{m}^{2}$, the reservoir is an effective reservoir; otherwise, it is a noneffective reservoir (see Fig. 4). Therefore, the porosity of $5 \%$ or more and the permeability of $0.15 \times 10^{-3} \mu \mathrm{m}^{2}$ or more can be used as the lower limit of the LX Block.
Table 1 Change in clastic composition from the Shiqianfeng to the Benxi formation

\begin{tabular}{|c|c|c|c|c|c|c|}
\hline \multirow[t]{2}{*}{ Position } & \multicolumn{2}{|c|}{ Quartz (\%) } & \multicolumn{2}{|c|}{ Feldspar (\%) } & \multicolumn{2}{|c|}{ Rock debris (\%) } \\
\hline & Average & Range & Average & Range & Average & Range \\
\hline Benxi formation & 37.7 & $35-71$ & 25.2 & $0-41$ & 36.5 & $20-65$ \\
\hline Taiyuan formation & 46.8 & $31-88$ & 18.0 & $0-49$ & 34.7 & $6-53$ \\
\hline Shanxi formation & 41.1 & $19-70$ & 17.1 & $0-34$ & 40.9 & $14-81$ \\
\hline Shihezi formation & 35.7 & $18-69$ & 28.1 & $4-44$ & 35.7 & $17-72$ \\
\hline Shiqianfeng formation & 30.0 & $12-51$ & 36.0 & $21-46$ & 35.0 & $21-55$ \\
\hline
\end{tabular}



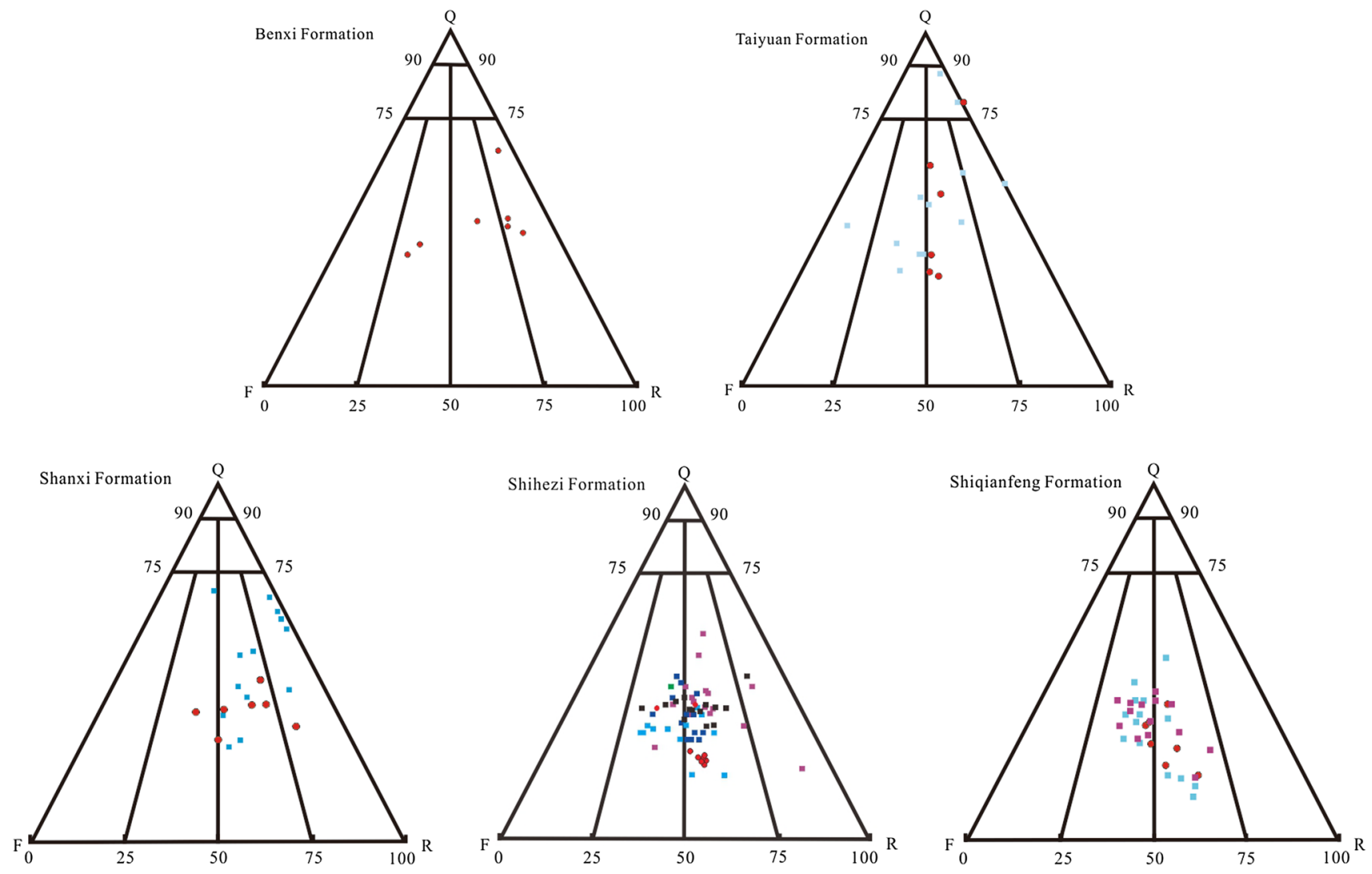

Fig. 1 Rock types of sandstone from the Shiqianfeng to the Benxi formation. Note: $Q$ quartz, $F$ feldspar, $R$ rock debris
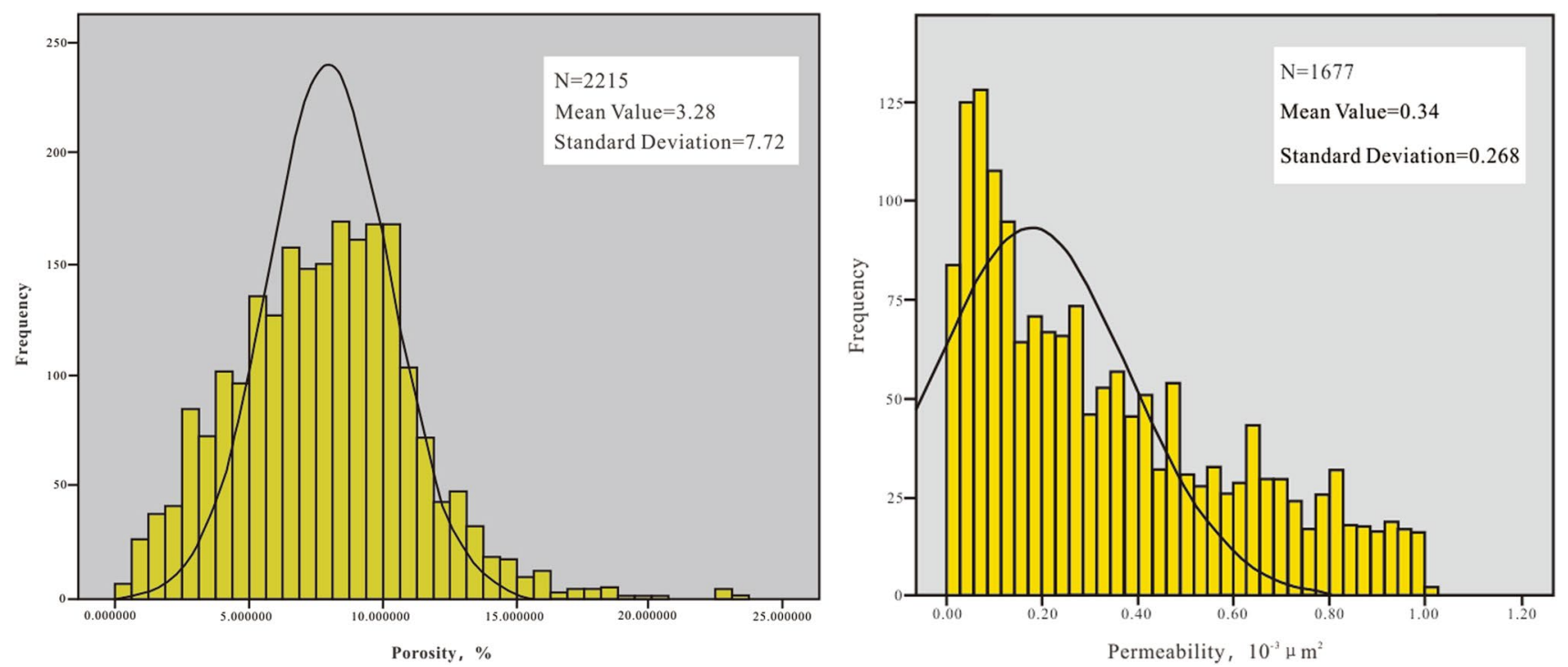

Fig. 2 Distribution histogram of the porosity and permeability in the LX Block

\section{Quantitative characterization of the gas log curve method}

The productivity is evaluated by the logging interpretation method to indirectly reflect the gas content in the reservoir according to the response relation of various parameters. Due to the strong heterogeneity, the low-resistance gas layer coexists with the high- and low-water-resistance layers in tight sandstone gas reservoirs, and it is difficult to truly reflect the gas content of the formation by 
Fig. 3 Pore types in the LX Block
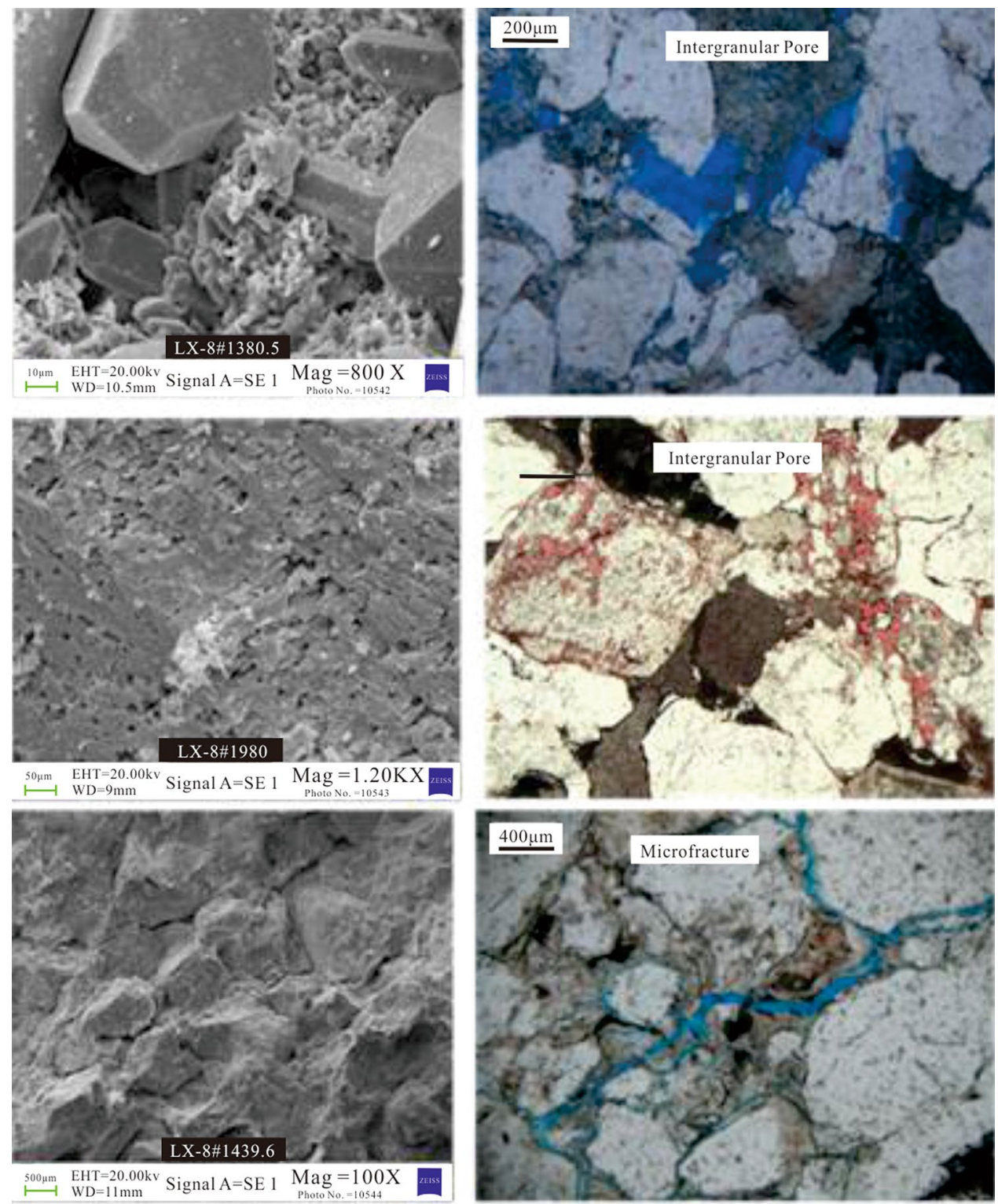

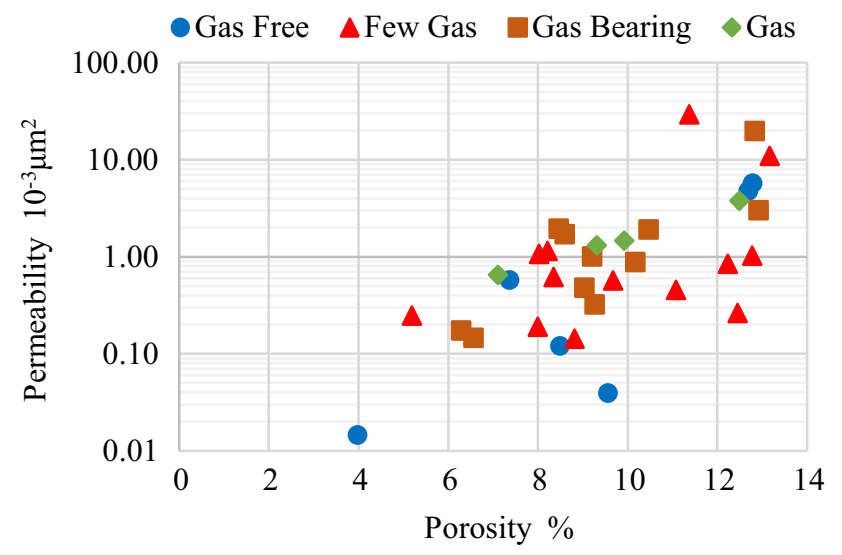

Fig. 4 Cross section of the porosity and permeability for the testing stages conventional logging interpretation methods. However, the mud logging interpretation method can directly detect the hydrocarbon content of the formation or drilling fluid and provide an accurate production capacity for the petroleum engineer. A limitation is also present in that there are different geological environments and engineering conditions of each well, so the total hydrocarbon value lacks comparability, which implies that it is difficult to directly analyze the reservoir physical properties and potential stimulation productivity. Thus, it is necessary to combine the logging interpretation method and mud logging evaluation method to improve the accuracy of the fracturing capacity prediction.

For tight sandstone gas reservoirs, due to the low oil-bearing, low absolute gas value and incomplete gas 


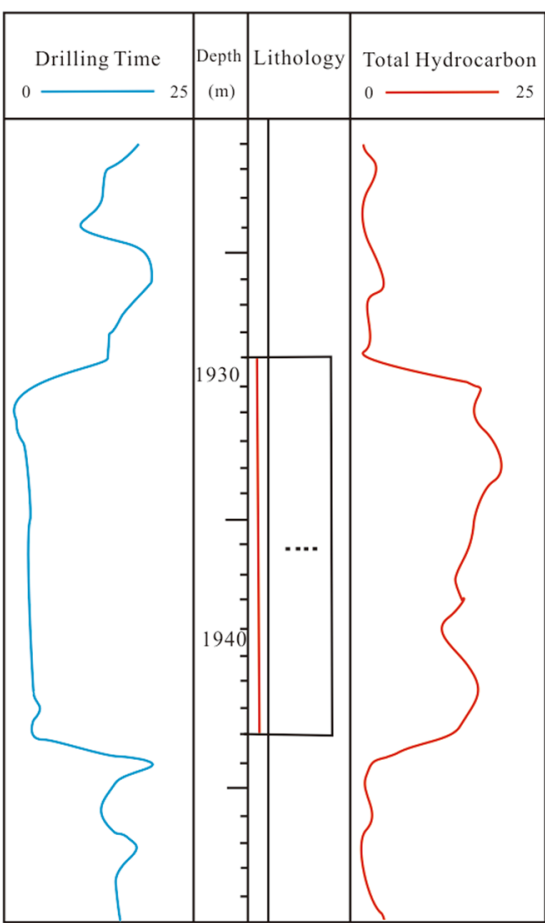

(a)

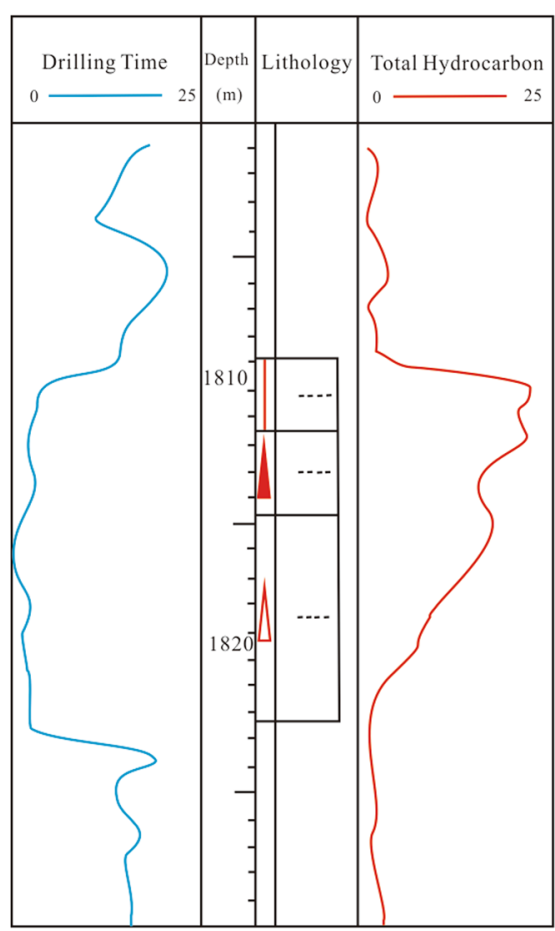

(d)

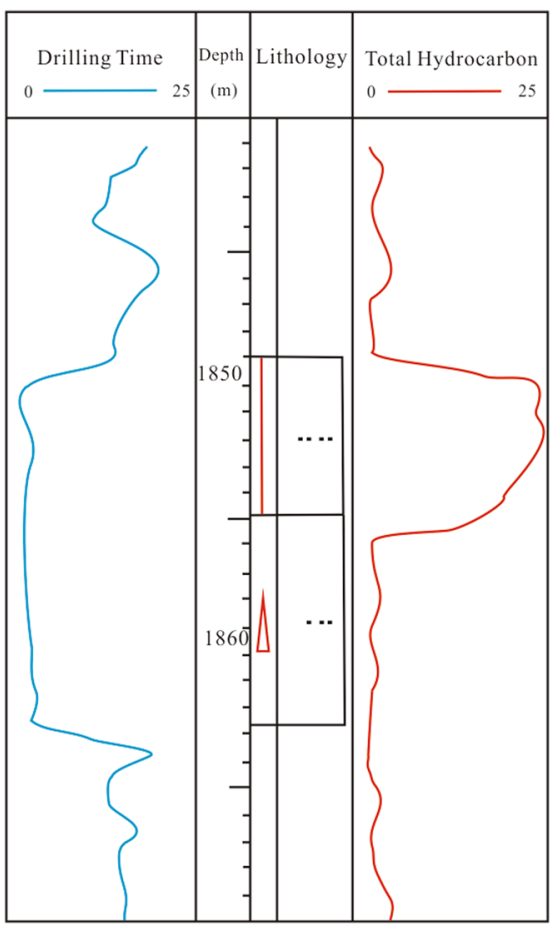

(b)

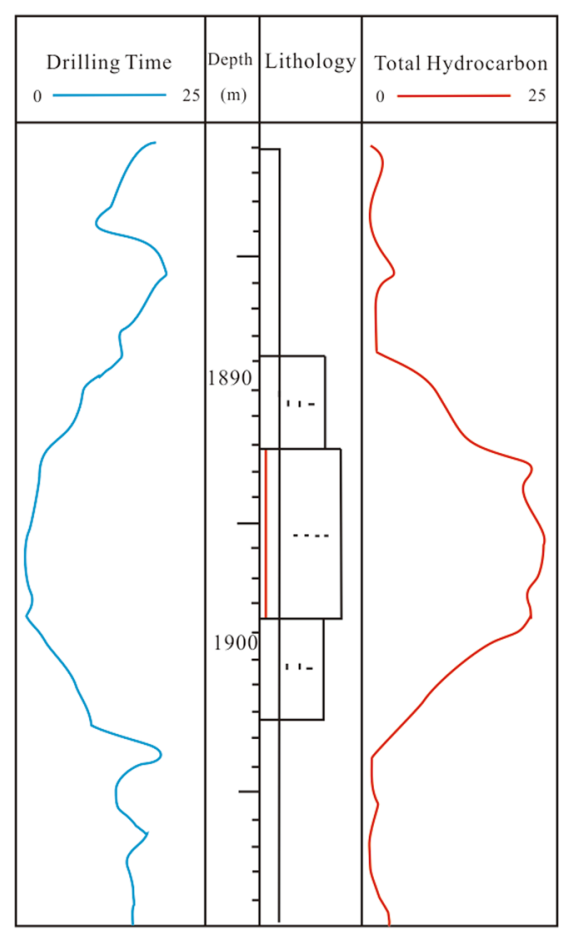

(e)

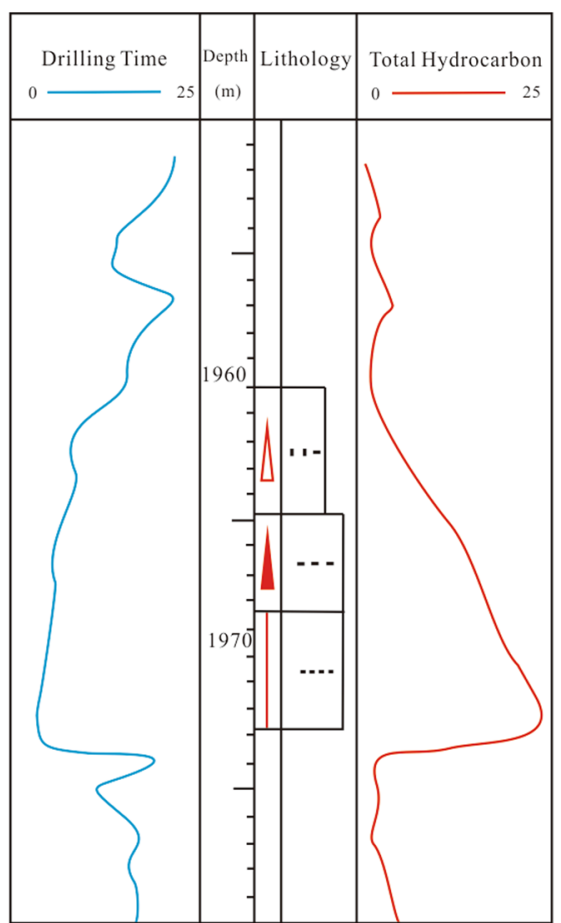

(c)

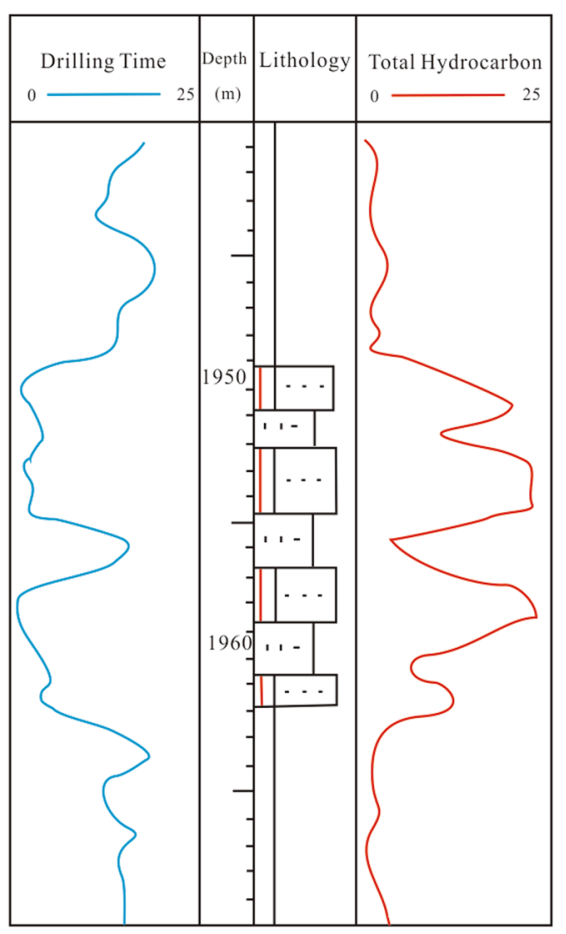

(f)

Fig. 5 Morphological characteristics of the total hydrocarbon curve. a Box, $\mathbf{b}$ half box, $\mathbf{c}$ triangle, $\mathbf{d}$ inverted triangle, $\mathbf{e}$ spike and $\mathbf{f}$ serration

components (such as gas layers and gas-water layers), conventional methods often fail to explain the gas-bearing properties of the formation. The morphological characteristics of the full hydrocarbon curve can be used to identify the properties of the reservoir fluid. Based on the analysis of 18 wells in the LX Block, the logging curve shapes of those gas zones are mostly box, followed by triangle, serration and half box shape. The gas layers corresponding to the single-spike gas logging curves are mostly dry or only nonindustrial gas layers, as shown in Fig. 5. 
Table 2 Relation of logging curve shapes and the productivity

\begin{tabular}{|c|c|c|}
\hline Serial no. & Criteria by well logging interpretation & Conclusion \\
\hline 1 & $\begin{array}{l}\text { The minimum value of M2R9 should be less than } 20 \Omega \mathrm{m} \text {, and the maximum value of sound wave should be } \\
\text { over } 70 \mu \mathrm{s} / \mathrm{ft} \text {, while the minimum value of the density should be under } 2.45 \mathrm{~g} / \mathrm{cm}^{3}\end{array}$ & High yield \\
\hline 2 & $\begin{array}{l}\text { The minimum value of M2R9 should be over } 20 \Omega \mathrm{m} \text {, and the maximum value of sound wave should be } \\
\text { higher than } 70 \mu \mathrm{s} / \mathrm{ft} \text {, while the minimum value of the density should be under } 2.45 \mathrm{~g} / \mathrm{cm}^{3}\end{array}$ & Gas \\
\hline 3 & $\begin{array}{l}\text { The minimum value of M2R9 should be } 20-25 \Omega \mathrm{m} \text {, and the maximum value of sound wave should be higher } \\
\text { than } 70 \mu \mathrm{s} / \mathrm{ft} \text {, while the minimum value of the density should be under } 2.45 \mathrm{~g} / \mathrm{cm}^{3}\end{array}$ & Suspicious gas \\
\hline 4 & $\begin{array}{l}\text { The minimum value of M2R9 should be less than } 20 \Omega \mathrm{m} \text {, and the maximum value of sound wave should be } \\
\text { less than } 70 \mu \mathrm{s} / \mathrm{ft} \text {, while the minimum value of the density should be over } 2.45 \mathrm{~g} / \mathrm{cm}^{3}\end{array}$ & Dry or gas-water \\
\hline 5 & $\begin{array}{l}\text { The minimum value of M2R9 should be over } 20 \Omega \mathrm{m} \text {, and the maximum value of sound wave should be less } \\
\text { than } 70 \mu \mathrm{s} / \mathrm{ft} \text {, while the minimum value of the density should be over } 2.45 \mathrm{~g} / \mathrm{cm}^{3}\end{array}$ & Gas or suspicious gas \\
\hline
\end{tabular}

Fuzzy mathematics is used to quantify the morphological characteristics of the gas logging curve. The average range of total hydrocarbon (ARTH), relative number of sawtooth (RNS) and variance are proposed to quantify the gas logging curve, and the automatic interpretation of each well is realized.

1. ARTH As the average range of the total hydrocarbon in the gas logging curve, which reflects the magnitude of the total hydrocarbon, the ARTH can be expressed as:

$$
\overline{\mathrm{ARTH}}=\sum_{i=1}^{n} \frac{r(i)}{n}
$$

where $r(i)$ is the total hydrocarbon value at point $\mathrm{i} ; \mathrm{n}$ is the number of the curve points.

2. RNS Since the thickness of each interval is different, the number of changes in the sign is used to reflect the RNS. First, a differential sequence is built, for the total hydrocarbon of gas logging curve, the differential sequence is: $T(2)-T(1), T(3)-T(2), \ldots, T(n)-T(n-1)$. Then, those signs change which the absolute value are greater than the mean value of $1 \%$ in the sequence can be counted as one sign change. The total number of sign changes is $L$, and the RNS can be written as:

$$
\mathrm{RNS}=L /(n-2)
$$

where $(n-2)$ is the maximum number of sign changes.

3. Variance $\Delta(i)$, which reflects the fluctuation of the entire curve:

$$
\Delta(i)=\frac{1}{n} \sum_{1}^{n}[r(i)-\bar{R}]
$$

\section{Qualitative analysis of the well logging interpretation}

The array-induced resistivity, sound wave, and density are closely related to the productivity. According to the
Table 3 Uniaxial compression test in LX Block

\begin{tabular}{lllll}
\hline Sample & Depth (m) & $\begin{array}{l}\text { Compressive } \\
\text { strength (MPa) }\end{array}$ & Poisson ratio & $\begin{array}{l}\text { Young } \\
\text { modulus } \\
(\mathrm{GPa})\end{array}$ \\
\hline D1-1 & 1854.43 & 84.35 & 0.2 & 18.49 \\
D2-1 & 1949.91 & 105.26 & 0.19 & 23.12 \\
D3-1 & 2062.68 & 66.65 & 0.23 & 15.93 \\
D1-1 & 1797.46 & 52.15 & 0.31 & 44.43 \\
D2-1 & 1799.32 & 48.59 & 0.27 & 57.75 \\
D3-1 & 1215.76 & 42.63 & 0.19 & 35.89 \\
D3-1 & 1283.54 & 40.63 & 0.17 & 19.43 \\
D3-2 & 1284.62 & 22.1 & 0.23 & 25.67 \\
D3-3 & 1286.34 & 49.35 & 0.16 & 32.62 \\
D6-1 & 1646.92 & 41.43 & 0.19 & 45.65 \\
D6-2 & 1649.3 & 48.35 & 0.16 & 54.52 \\
D6-3 & 1656.22 & 48.6 & 0.17 & 37.16 \\
\hline
\end{tabular}

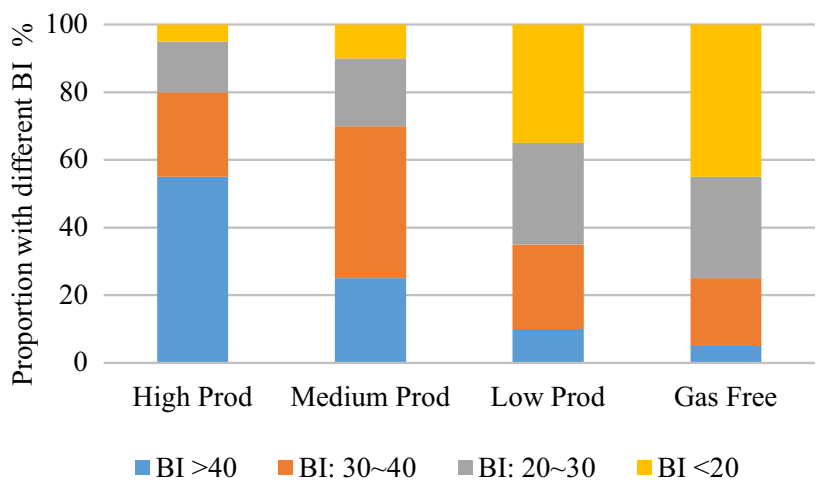

Fig. 6 Relationship between the BI and productivity

matching relation analysis between the gas test results and the morphological characteristics of the well logging curves, it can be divided into 5 categories (see Table 2 for details). In particular, the shape of the logging curve can only qualitatively reflect the gas production capacity of the reservoir. 
Table 4 Classification assignment of morphological characteristics in the LX Block

\begin{tabular}{lllll}
\hline $\begin{array}{l}\text { Morphological } \\
\text { characteristics }\end{array}$ & Serial no. & $\begin{array}{l}\text { Production } \\
\left(\times 10^{4} \mathrm{~m}^{3} / \mathrm{d}\right)\end{array}$ & Assignment & $\begin{array}{l}\text { Weight } \\
\text { coeffi- } \\
\text { cient }\end{array}$ \\
\hline Box & 1 & $<2$ & 0.8 & 0.5 \\
Semi-Box & 2 & $\geq 2$ & 1.0 & \\
& 3 & $<2.5$ & 0.6 & 0.5 \\
Triangle & 4 & $\geq 2.5$ & 0.8 & \\
Serration & 5 & - & 0.9 & 0.5 \\
& 6 & $<0.8$ & 0.7 & 0.5 \\
Spike & 7 & $\geq 0.8$ & 0.9 & \\
& 8 & $<0.8$ & 0.7 & 0.5 \\
Others & 9 & $\geq 0.8$ & 0.9 & \\
\hline
\end{tabular}

To obtain accurate productivity evaluation results, it can be combined with the gas log curve method, and the well logging analysis results can be used to correct the final quantitative characterization conclusion.

Based on the above analysis, the gas log curve method can be used to determine the morphological characteristics, and the gas productivity can be quantitatively calculated. The well logging curve analysis can offer a trend conclusion of the gas capacity and find the sweet spots that satisfy the characteristics of the gas logging curve.

\section{Interpretation model and productivity calculation}

The interpretation model is a comprehensive reflection of the actual production performance. Considering only one factor alone will cause uncertainty in the production prediction. Therefore, the morphological characteristics of the logging curves, mud logging curves and key geological parameters must be considered. The fuzzy mathematics method is used to assign each factor, and the final calculation model is established.

The rock brittleness is an intrinsic property when rock fails under stress. It is related to the mineral composition and
Table 6 Classification assignment of the BI in the LX Block

\begin{tabular}{llll}
\hline Level & Criterion & Assignment & $\begin{array}{l}\text { Weight } \\
\text { coefficient }\end{array}$ \\
\hline A & BI $>40.0$ & 1 & 0.3 \\
B & $\mathrm{BI} \in[30,40]$ & 0.8 & 0.3 \\
C & $\mathrm{BI} \in[20,30]$ & 0.6 & 0.3 \\
$\mathrm{D}$ & $\mathrm{BI}<20$ & 0.1 & 0.2 \\
\hline
\end{tabular}

The unit of the BI is \%

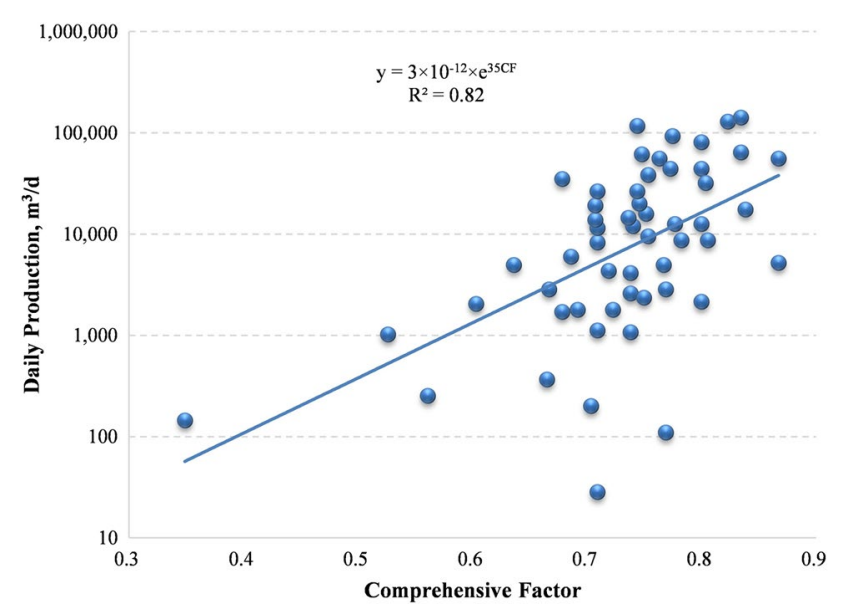

Fig. 7 Relationship between the $\mathrm{CF}$ and productivity

mechanical properties of the rock. The brittleness index (BI) characterizes the degree of transient variation of the rock before it breaks and is closely related to the complexity of the fractures after hydraulic fracturing, which is one of the key parameters for volume fracturing in low-permeability tight reservoirs. According to Table 1, the tight sand formation in the studied area are featured by high contents of brittle minerals. Similar to the shale reservoir, BI has close relationship with the final production, especially after hydraulic fracturing. A higher Young's modulus and a smaller Poisson's ratio correspond to a higher brittleness index. In practical applications, the dynamic rock mechanics parameters are
Table 5 Classification assignment of physical properties in the LX Block

\begin{tabular}{llll}
\hline Level & Criterion & Assignment & $\begin{array}{l}\text { Weight } \\
\text { coefficient }\end{array}$ \\
\hline A & Porosity $\in[20.0,25.0]$ & 1 & 0.2 \\
B & Porosity $\in[15.0,20.0]$, permeability $>50.0$ & 0.9 & 0.2 \\
C & Porosity $\in[10.0,15.0]$, permeability $\in[1.0,50.0]$ & 0.6 & 0.2 \\
D & Porosity $\in[5.0,10.0]$, permeability $\in[0.1,1.0]$ & 0.5 & 0.2 \\
E & Porosity $<5$ or permeability $\leq 0.1$ & 0 & 0.2 \\
F & Average porosity $\geq 25$ & 0 & 0.2 \\
\hline
\end{tabular}

The unit of the porosity is $\%$ and permeability is $10^{-3} \mu \mathrm{m}^{2}$ 


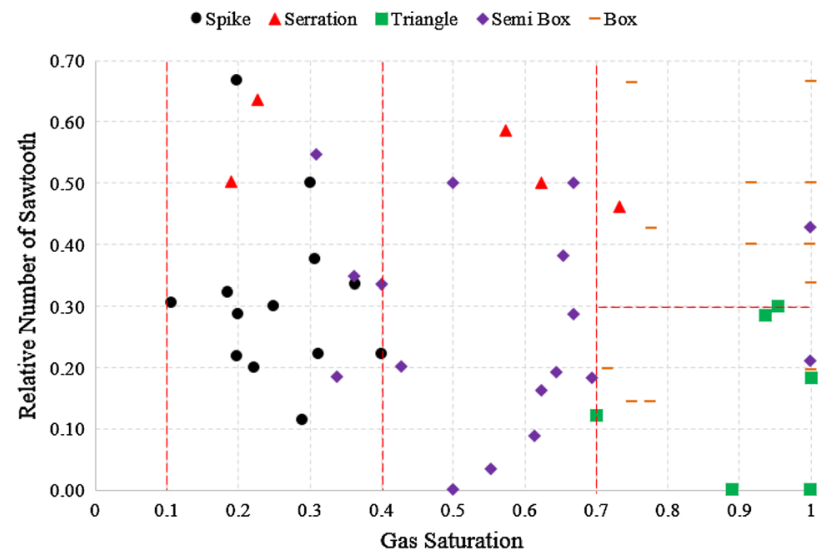

Fig. 8 Relation diagram between gas saturation and RNS

Table 7 Productivity characteristics in LX Block

\begin{tabular}{lllll}
\hline Class & $\begin{array}{l}\text { Resistivity } \\
(\Omega \mathrm{m})\end{array}$ & Density $\left(\mathrm{g} / \mathrm{cm}^{3}\right)$ & $\begin{array}{l}\text { Sound wave } \\
(\mu \mathrm{s} / \mathrm{ft})\end{array}$ & Prediction \\
\hline I & $<20$ & $<2.45$ & $>70$ & High \\
II & $>20$ & $<2.45$ & $>70$ & Medium \\
III & $>20$ & $<2.45$ & $>70$ & Low \\
IV & $>20$ & $>2.45$ & $<70$ & Dry \\
\hline
\end{tabular}

often calculated based on the well logging data (Rickman et al. 2008). The BI is selected for assignment in this study.

$E_{\mathrm{BRIT}}=\left[\left(E_{c}-E_{c \min }\right) /\left(E_{c \max }-E_{c \min }\right)\right]$

$v_{\mathrm{BRIT}}=\left[\left(v_{c}-v_{c \max }\right) /\left(v_{c \min }-v_{c \max }\right)\right]$

$\mathrm{BI}=\left(E_{\mathrm{BRIT}}+v_{\mathrm{BRIT}}\right) / 2$

where $E_{\mathrm{BRIT}}$ is the normalized Young's modulus, dimensionless; $E_{c}$ is the dynamic Young's modulus of the investigated formation, dimensionless; $E_{c \max }$ and $E_{c \min }$ are the dynamic maximum and minimum Young's modulus of the investigated formation, respectively, dimensionless; $v_{\text {BRIT }}$ is the normalized Poisson's ratio, dimensionless; $v_{c}$ is the dynamic Poisson's ratio of the investigated formation, dimensionless; and $v_{c \max }$ and $v_{c \min }$ are the maximum and minimum Poisson's ratios of the investigated formation, respectively, dimensionless. BI is the brittle index of the rock.

Table 3 is some of the uniaxial compression test results in LX Block. According to the trial calculation of 108 intervals, the BI of the test gas zone is mostly distributed in the range of $20-30 \%$; the zones with BI above $40 \%$ have a higher production than the dry layers. As shown in Fig. 6, in the high-yield gas layers, 55\% of the layers have a BI above $40 \%$; in the medium-production gas layers, the BI is approximately $30-40 \%$. The BI of the lowproduction gas layers and nonproductive gas layers are significantly lower than those of medium- or high-production gas layers.

In summary, we first set the weight coefficient of the morphological characteristics of the gas logging curve as 0.5. Then, the morphological characteristics are divided into 10 categories, and the assignment of each category can be checked in Table 4.

We set the weight coefficient of the $\mathrm{BI}$ as 0.3 and divide the brittleness into 4 levels.

Assuming that the morphological characteristics, physical properties and BI of a gas stage are assigned as a, b, and c, respectively, the comprehensive factor $(\mathrm{CF})$ can be expressed as:

$\mathrm{CF}=0.5 a+0.2 b+0.3 c$

The value of $a, b$ and $c$ can be obtained from Tables 4, 5, 6 . A correlation analysis has been made based on the calculation of 152 gas stages. According to Fig. 7, the result shows a good correlation between the $\mathrm{CF}$ and productivity, where the correlation degree is $82 \%$. Based on the result of the correlation analysis, the prediction model of productivity can be written as:

$Q=3 \times 10^{-12} \times e^{35 \mathrm{CF}}$

\section{Results analysis and discussion}

\section{Productivity prediction analysis based on the gas logging curve characteristic}

The thickness of the target zone is $5.0-15.0 \mathrm{~m}$ in the LX Block. It is a typical tight gas reservoir, where the average porosity is less than $10 \%$, and the average permeability is less than $1 \times 10^{-3} \mu \mathrm{m}^{2}$. According to the morphological analysis of the well logging curve and mud logging curve, most of the high-production zones are (semi) box-shaped, while the low-production zones have serration and spike shapes.

As shown in Fig. 8, the curves with the spike shape are mostly distributed in the left-middle area of the diagram (low gas saturation and low RNS), serration-shaped curves are in the upper area (high RNS), triangle-shaped curves are in the bottom right area (high saturation and low RNS), semi-box-shaped curves are scattered in the middle of the graph, and box-shaped curves are spread in the top right area (high gas saturation and high RNS). The morphological characteristics of the gas logging curves can be well distinguished by the relationship between gas saturation and 


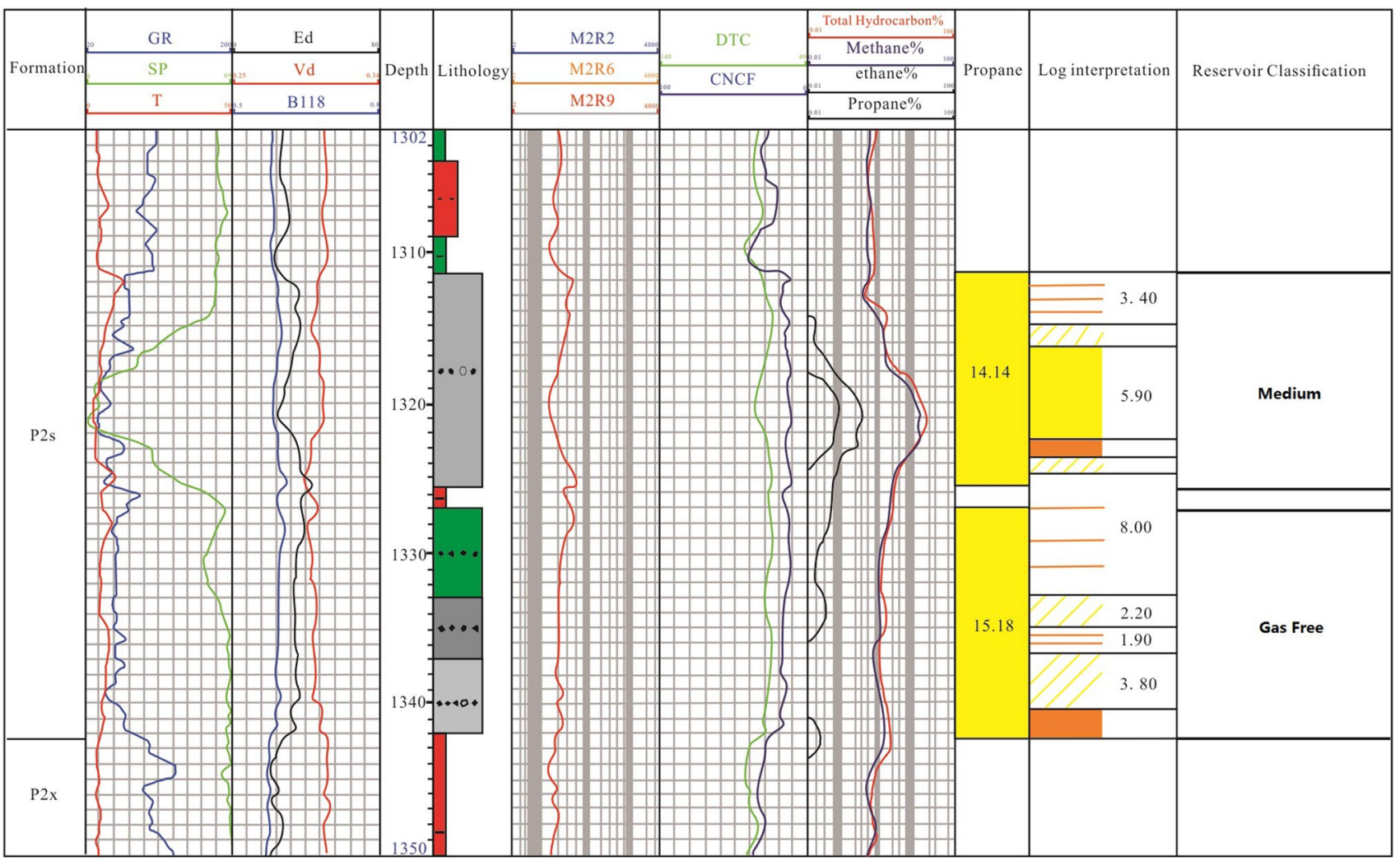

Fig. 9 Interpretation results of the Shihezi formation of well M-1

RNS, which can be considered the basis for identifying the gas-bearing zones.

\section{Productivity prediction analysis based on the well logging curve characteristic}

According to the characteristics of the well logging curve, the reservoir production performance of the study area can be divided into four categories. Table 7 shows the productivity characteristics in the LX Block.

The resistivity curve of the class-I reservoir presents a negative abnormal on low value, the minimum arrayinduced resistivity (AIR) is under $20 \Omega \mathrm{m}$, and the density is lower than $2.45 \mathrm{~g} / \mathrm{cm}^{3}$. The acoustic time is above $70 \mu \mathrm{s} / \mathrm{m}$. This type of reservoir is expected to have high production potential.

The resistivity curve of the class-II reservoir presents a positive abnormal on high value, the minimum AIR is above $20 \Omega \mathrm{m}$, and the minimum density is under $2.45 \mathrm{~g} / \mathrm{cm}^{3}$. The acoustic time is above $70 \mu \mathrm{s} / \mathrm{m}$. This type of reservoir is expected to have medium production potential.

The resistivity curve of the class-III reservoir presents a positive abnormal on low value, the minimum AIR is under $20 \Omega \mathrm{m}$, and the density is higher than $2.45 \mathrm{~g} / \mathrm{cm}^{3}$.
The acoustic time is under $70 \mu \mathrm{s} / \mathrm{m}$. This type of reservoir is expected to have low production potential.

The resistivity curve of the class-IV reservoir presents a positive abnormal on high value, the minimum AIR is under $20 \Omega \mathrm{m}$, and the density is above $2.45 \mathrm{~g} / \mathrm{cm}^{3}$. The acoustic time is under $70 \mu \mathrm{s} / \mathrm{m}$. This type of reservoir is expected to have dry gas potential.

\section{Discussion}

With well M-1 as an example, the target zone is $1311.5-1325.5 \mathrm{~m}$, the average pore radius is $0.218-0.586 \mu \mathrm{m}$, and the distribution pattern of pore throat is unimodal type. The average porosity of the formation is approximately $8 \%$, and the permeability is $0.5 \times 10^{-3} \mu \mathrm{m}^{2}$. According to the conclusion of the reservoir quality limit in 3.1.4, well M-1 has exploration value.

As shown in Fig. 9, the reservoir gas saturation is 0.88, and the RNS is 0.47 , which belongs to the box shape with medium production. According to Tables 2 and 6, the class-I reservoir is defined by the well logging interpretation results, which belongs to the type of multiproductive gas with high production. 
Table 8 Coincidence rate between the predicted productivity and gas testing results

\begin{tabular}{|c|c|c|c|c|c|c|}
\hline Well & $\begin{array}{l}\text { Permeability } \\
\left(\times 10^{-3} \mu \mathrm{m}^{3}\right)\end{array}$ & Porosity (\%) & Shape & Evaluation & $\begin{array}{l}\text { Gas prod } \\
\left(\times 10^{4} \mathrm{~m}^{3}\right)\end{array}$ & Results \\
\hline$X-1$ & 1.26 & 4.82 & Triangle & $\mathrm{F}$ & 0 & Match \\
\hline$X-2$ & 0.48 & 8.99 & Serration & $\mathrm{L}$ & 2.67 & Match \\
\hline$X-3$ & 0.17 & 5.44 & Spike & $\mathrm{F}$ & 0 & Match \\
\hline$X-4$ & 0.28 & 12.43 & Spike & $\mathrm{F}$ & 0 & Match \\
\hline$X-5$ & 1.82 & 4.49 & Triangle & $\mathrm{L}$ & 1.65 & Match \\
\hline$X-6$ & 0.62 & 9.69 & Spike & $\mathrm{L}$ & 0 & Not match \\
\hline$X-7$ & 1.13 & 7.99 & Spike & $\mathrm{L}$ & 0 & Not match \\
\hline LX-1 & 1.29 & 9.28 & Serration & M & 9.64 & Match \\
\hline LX-2 & 1.41 & 3.37 & Spike & $\mathrm{L}$ & 0.17 & Not match \\
\hline LX-3 & 0.26 & 5.20 & Triangle & $\mathrm{F}$ & 0.02 & Match \\
\hline LX-4 & 0.67 & 8.34 & Triangle & $\mathrm{F}$ & 0.01 & Match \\
\hline LX-5 & 0.16 & 8.82 & Spike & $\mathrm{F}$ & 0.02 & Match \\
\hline LX-6 & 0.50 & 3.07 & Serration & $\mathrm{F}$ & 0 & Match \\
\hline LX-7 & 1.32 & 5.49 & Spike & $\mathrm{L}$ & 1.65 & Match \\
\hline LX-8 & 0.73 & 7.96 & Triangle & $\mathrm{L}$ & 1.35 & Match \\
\hline LX-9 & 0.93 & 8.99 & Box & M & 8.53 & Match \\
\hline LX-10 & 0.34 & 6.28 & Spike & $\mathrm{L}$ & 1.64 & Match \\
\hline LX-11 & 1.51 & 9.37 & Box & $\mathrm{H}$ & 12.37 & Match \\
\hline LX-12 & 0.56 & 7.20 & Triangle & $\mathrm{L}$ & 0.02 & Not match \\
\hline LX-101 & 0.76 & 7.34 & Semi-Box & M & 1.38 & Not match \\
\hline LX-102 & 0.49 & 6.28 & Serration & $\mathrm{F}$ & 0.02 & Match \\
\hline LX-103 & 0.36 & 8.22 & Spike & $\mathrm{F}$ & 0 & Match \\
\hline LX-104 & 0.57 & 7.07 & Serration & $\mathrm{F}$ & 0.01 & Match \\
\hline MX-1 & 0.20 & 7.92 & Spike & $\mathrm{F}$ & 0 & Match \\
\hline MX-2 & 0.73 & 1.72 & Box & F & 0 & Match \\
\hline MX-3 & 1.01 & 9.15 & Box & M & 4.20 & Match \\
\hline MX-4 & 0.80 & 5.68 & Spike & $\mathrm{L}$ & 0 & Not match \\
\hline MX-5 & 0.92 & 4.18 & Box & $\mathrm{F}$ & 0 & Match \\
\hline MX-6 & 0.90 & 12.23 & Spike & $\mathrm{F}$ & 0 & Match \\
\hline MX-7 & 0.33 & 9.24 & Box & M & 3.64 & Not match \\
\hline MX-8 & 1.90 & 8.49 & Semi-Box & $\mathrm{L}$ & 0.60 & Match \\
\hline $\mathrm{T}-1$ & 3.07 & 12.89 & Box & M & 3.31 & Match \\
\hline $\mathrm{T}-2$ & 1.14 & 7.97 & Spike & $\mathrm{F}$ & 0 & Match \\
\hline $\mathrm{T}-3$ & 1.11 & 12.73 & Triangle & F & 0 & Match \\
\hline $\mathrm{T}-4$ & 0.12 & 8.49 & Spike & $\mathrm{F}$ & 0 & Match \\
\hline $\mathrm{T}-5$ & 2.91 & 13.76 & Box & M & 5.70 & Match \\
\hline $\mathrm{H}-1$ & 0.71 & 7.08 & Box & $\mathrm{H}$ & 11.95 & Match \\
\hline $\mathrm{H}-2$ & 0.90 & 10.12 & Box & $\mathrm{L}$ & 1.79 & Match \\
\hline $\mathrm{H}-3$ & 0.15 & 6.58 & Triangle & $\mathrm{L}$ & 1.19 & Match \\
\hline $\mathrm{H}-4$ & 1.89 & 10.48 & Serration & $\mathrm{L}$ & 0.89 & Match \\
\hline H-5 & 1.69 & 8.61 & Box & $\mathrm{L}$ & 2.09 & Match \\
\hline H-6 & 0.18 & 6.31 & Serration & $\mathrm{L}$ & 0.83 & Match \\
\hline
\end{tabular}

In evaluation column, $F$ gas free, $L$ low, $M$ medium, $H$ high
The CF is 0.83 based on the calculation of formula (7), and the predicted production is $12.40 \times 10^{4} \mathrm{~m}^{3} / \mathrm{d}$. Thus, according to the multifactor analysis, this layer is defined as high yield, and the field gas test result is $12.91 \times 10^{4} \mathrm{~m}^{3} / \mathrm{d}$.
The judgment results are consistent with the actual gas production capacity.

As shown in Table 8, the gas testing results of 42 wells were analyzed. The low-production or dry gas wells 
occupied $79.5 \%$, while the medium- or high-production layers only occupied $20.5 \%$. The predicted coincidence rate of production and nonproduction was $84.1 \%$. Thus, the new evaluation approach can satisfy the demands of field operation, and the prediction results have a high reference value.

\section{Conclusion}

To accurately evaluate the productivity potential of tight gas wells, this study takes the LX Block of Ordos Basin in China as the object. Based on the core lab test and field data of the study area, the reservoir physical properties in the LX Block were analyzed. The change rules of gas bearing were determined using the method of logging curve shape theory. The effect of rock brittleness on the daily production was considered, and the fuzzy mathematics method was used to assign the influencing parameters. The main conclusions are as follows:

1. The gas logging curve can be divided into six types of shapes: box shape, semi-box shape, triangle shape, serration shape, clock shape and spike shape. Each curve shape has a close relationship with the gas content. The RNS can be used as the quantitative characterization parameter of these morphological characteristics.

2. The potential productivity of tight sand gas can be divided into four categories according to the comprehensive methodology of well logging and mud logging. The potential productivity of the target zone in the LX Block is poor, the first-class and second-class reservoirs only occupy $16.75 \%$, and hydraulic fracturing is required for the reservoir stimulation.

3. The productivity prediction model with comprehensive interpretation of well logging and mud logging can forecast the production of the tight gas reservoir. The coincidence rate between the prediction and actual situation reaches $84.1 \%$.

To increase the accuracy of the evaluation method on tight sandstone productivity, further study on other gas logging curves such as methane, ethane and propane is recommended as the next step.

Acknowledgements This study was funded by the Project of the Key Laboratory of Well Stability and Fluid \& Rock Mechanics in Oil and Gas Reservoir of Shaanxi Province, Xi' an Shiyou University under Grant No. WSFRM20190302001, the Research Project of the Hubei Provincial Department of Education under Grant No. Q20181307, the Project of Shandong Provincial Key Laboratory of Depositional Mineralization and Sedimentary Mineral under Grant No. DMSM2019001, the National Science and Technology Major Project under Grant No. 2016ZX05061-009.
Open Access This article is licensed under a Creative Commons Attribution 4.0 International License, which permits use, sharing, adaptation, distribution and reproduction in any medium or format, as long as you give appropriate credit to the original author(s) and the source, provide a link to the Creative Commons licence, and indicate if changes were made. The images or other third party material in this article are included in the article's Creative Commons licence, unless indicated otherwise in a credit line to the material. If material is not included in the article's Creative Commons licence and your intended use is not permitted by statutory regulation or exceeds the permitted use, you will need to obtain permission directly from the copyright holder. To view a copy of this licence, visit http://creativecommons.org/licenses/by/4.0/.

\section{References}

Abdelmoneam ER, Matthew WT, Aria L et al (2019) Lithofacies control on reservoir quality of the viola limestone in Southwest Kansas and unsupervised machine learning approach of seismic attributes facies-classification. Pure Appl Geophys 176(10):4297-4308

Anand V (2017) Novel methodology for accurate resolution of fluid signatures from multi-dimensional NMR well-logging measurements. J Magn Reson 276:60-68

Cao N, Lei G (2019) Stress sensitivity of tight reservoir during pressure loading and unloading process. Pet Explor Dev 46(1):1-7

Chaki S, Routray A, Mohanty WK (2018) Well-Log and seismic data integration for reservoir characterization: a signal processing and machine-learning perspective. IEEE Signal Process Mag 35(2):72-81

Fall A, Eichhubl P, Bodnar RJ et al (2015) Natural hydraulic fracturing of tight-gas sandstone reservoirs, Piceance Basin, Colorado. Geol Soc Am Bull 127(1-2):61-75

Fang Z, Liu YW, Yang DX et al (2018) Real-time hydrogen mud logging during the Wenchuan earthquake fault scientific drilling project (WFSD), holes 2 and 3 in SW China. Geosci $J$ 22(3):453-464

Guo CH, Xu JC, Wei MZ et al (2015) Experimental study and numerical simulation of hydraulic fracturing tight sandstone reservoirs. Fuel 159:334-344

Kulga B, Artun E, Ertekin T (2017) Development of a data-driven forecasting tool for hydraulically fractured, horizontal wells in tight-gas sands. Comput Geosci 103:99-110

Liu ZX, Wang XZ, Li JS et al (2016) New technique in the identification of water-gas interface in low permeability gas reservoirs from core data. Pet Drill Tech 44(6):88-92

Liu D, Xiao L, Li JR et al (2018a) Method of predicting tight Gas deliverability from conventional well logging data based on experimental simulation. Arab J Sci Eng 43(5):2615-2623

Liu SY, Zhang YX, Lou YS et al (2018b) Novel evaluation approach for shale brittleness index. DYNA 93(4):442-447

Montgomery JB, O'Sullivan FM (2017) Spatial variability of tight oil well productivity and the impact of technology. Applied Energy 195(C):344-355

Rickman R, Mullen M, Petre E et al (2008) A practical use of shale petrophysics for stimulation design optimization: all shale plays are not clones of the Barnett Shale. In: SPE annual technical conference and exhibition, Denver, Colorado, USA

Sakhaee-Pour A (2017) Decomposing J-function to account for the pore structure effect in tight gas sandstones. Transp Porous Media 116(2):453-471

Sander R, Pan ZJ, Connell LD (2017) Laboratory measurement of low permeability unconventional gas reservoir rocks: a review of experimental methods. J Nat Gas Sci Eng 37:248-279 
Sarhan MA, Basal AMK, Ibrahim IM (2017) Seismic and well logging interpretation for evaluation of the lower Bahariya reservoir, southwest Qarun (SWQ) Field, Gindi Basin, Egypt. Mar Geophys Res 38(3):271-290

Schmitt M, Fernandes CP, Wolf FG et al (2015) Characterization of Brazilian tight gas sandstones relating permeability and Angstrom-to micron-scale pore structures. J Nat Gas Sci Eng 27(2):785-807

Shao XH, Pang XQ, Li H et al (2017) Fractal analysis of pore network in tight gas sandstones using NMR method: a case study from the Ordos Basin, China. Energy Fuels 31(10):10358-10368

Song MS, Liu Z, Zhang XC et al (2019) Fracability evaluation of tight sandstone reservoirs based on improved entropy analytic hierarchy process: taking the Jurassic reservoirs of Well Z109 in the Junggar Basin as an example. J Geomech 25(4):509-517

Sun JM (2013) Coalbed methane and shale gas evaluation based on new seven related logging goals. Well Logging Technol 37(5):457-465

Umrao RK, Sharma LK, Singh R et al (2018) Determination of strength and modulus of elasticity of heterogenous sedimentary rocks: an ANFIS predictive technique. Measurement 126:194-201
Wu H, Ji YL, Liu R et al (2017a) Insight into the pore structure of tight gas sandstones: a case study in the Ordos Basin, NW China. Energy Fuels 31(12):13159-13178

Wu H, Chen P, Fan XY et al (2017b) Research on rapid identification and evaluation technology for gas formation during underbalanced drilling. Geofluids 11:1-8

Xiao DS, Lu SF, Yang JX et al (2017) Classifying multiscale pores and investigating their relationship with porosity and permeability in tight sandstone gas reservoirs. Energy Fuels 31(9):9188-9200

Zou CN, Zhai GM, Wang HJ et al (2015) Formation, distribution, potential and prediction of global conventional and unconventional hydrocarbon resources. Pet Explor Dev 42(1):13-25

Publisher's Note Springer Nature remains neutral with regard to jurisdictional claims in published maps and institutional affiliations. 